\title{
Nutrition Research and Human Disease: A Critical Appraisal of Mechanistic Research, Cohort Studies, and Randomized Trials
}

\section{Temple $\mathrm{NJ}^{*}$}

\author{
Centre for Science, Athabasca University, Athabasca, Canada
}

*Corresponding author: Temple NJ, Centre for Science, Athabasca University, Athabasca, Canada, Fax: 780 675 6186, Tel: 780450 0167, E-mail: normant@athabascau.ca

Citation: Temple NJ (2015) Nutrition Research and Human Disease: A Critical Appraisal of Mechanistic Research, Cohort Studies, and Randomized Trials. J Nutr Health Sci 2(1): 101. doi: 10.15744/23939060.1.405

\section{Received Date: November 03, 2014 Accepted Date: February 03, 2015 Published Date: February 09, 2015}

\begin{abstract}
A major part of nutrition research consists of the investigation of how food components affect the biochemical and physiological processes within the body. The rationale is that this mechanistic research will lead to a fuller understanding of disease etiology thereby generating information of practical value for the treatment and prevention of disease. More direct approaches to understanding dietdisease relationships are based on cohort studies and randomized controlled trials (RCTs). This paper critically examines examples of diet-disease relationships so as to determine which research approaches have been most productive. Areas covered include several foods (such as sugar-sweetened beverages, fish, meat, and fruit), several nutrients (such as fat, sodium, and selenium), and several diseases/disorders (hypertension, obesity, cancer, and coronary heart disease). This analysis reveals that most of our information of practical value has come from cohort studies and RCTs but relatively little has come from mechanistic research. It follows, therefore, that top priorities for nutrition research should be the carrying out of more cohort studies and RCTs. This is then discussed with reference to research on phytochemicals. However, mechanistic research has been of value in particular areas. This occurs where disease processes involve simple mechanisms; examples include several metabolic disorders with a genetic basis (such as lactose intolerance) and deficiencies of various vitamins and minerals.
\end{abstract}

Keywords: Cohort studies; Randomized controlled trials; Mechanistic research; Phytochemicals

\section{Introduction}

For many decades researchers have followed the strategy that in order to achieve a fuller understanding of nutrition, it is necessary to study the biochemical and physiological action of the huge numbers of chemicals in food, and thence learn the mechanisms by which they protect health or increase risk of disease [1-3]. This strategy, often known as reductionism, has revealed a great deal about the role played in the body by vitamins, minerals, and many other substances, and why deficiencies of them lead to specific symptoms. However, this mechanistic strategy has achieved little success in recent decades in terms of generating information that is of practical value with regard to nutrition and human health [1-3]. The reason for this is that because of the great complexity of the human body it is extraordinarily difficult to properly understand the exact details of the pathways leading to disease. Even with relatively "simple" disorders, such as hypertension, obesity, and type 2 diabetes, there are multiple pathways involved and the story of each disorder becomes steadily more complex as new discoveries are made. There is an additional reason for the poor success of mechanistic research as applied to nutrition: foods contain thousands of separate substances and this leads to vast numbers of possible interactions.

We can illustrate this with the example of olive oil and coronary heart disease (CHD). Much epidemiological (observational) evidence indicates an inverse association between consumption of a Mediterranean diet and risk of CHD. One component of this diet suspected of playing an important role is olive oil. A researcher decides to study the effects of olive oil on the body's biochemical and physiological processes focusing on those that may indicate whether olive oil affects the risk of CHD and, if it does, how this may occur. Her research team therefore investigates how the amount of olive oil in the diet affects a wide array of body mechanisms, including the following: the levels of various types of prostaglandins (and related substances), various mechanisms related to inflammatory processes, receptors on the cell surfaces of different tissues and how they respond to insulin (and other hormones), the processes related to atherogenesis within the artery wall, the mechanisms that regulate lipogenesis, and the control of energy balance (including the various hormones involved and their interactions with brain areas that regulate the appetite). Fully exploring how olive oil affects these processes will likely necessitate 20 or 30 years of total commitment by the 20 members of her research team (and tens of millions of dollars of NIH funding). However, major areas have not yet been touched, including: mechanisms involved in CHD that have not yet been discovered, interactions between olive oil and the many other components of the Mediterranean diet (such as the myriad phytochemicals present in wine and fruit), and the control systems operating at the genetic level that switch the systems off and on. 
A competing strategy is based on directly studying whether different variables related to diet (as well as other aspects of lifestyle) influence disease outcomes. Major types of research in this area are epidemiology (including cohort studies) and randomized controlled trials (RCTs). Applying this to the above example, researchers would carry out two major types of study: (1) cohort studies in order to determine whether dietary intake of olive oil is related to risk of CHD, and (2) RCTs in order to determine whether supplements of olive oil affect actual risk of CHD.

\section{Lessons from Research on Diet and Disease}

The following examples illustrate how most of our information of practical value in the field of nutrition and disease has come from cohort studies and RCTs, but relatively little from mechanistic research. Other types of epidemiological studies besides cohort studies have also been of much value (i.e., case-control studies and cross-sectional studies) but are not included here as they generally produce findings of less reliability than cohort studies [4]. Population comparisons (ecological studies) are mentioned briefly as they can add strength to the findings coming from cohort studies and RCTs.

\section{Example 1: Sodium intake and blood pressure}

Population comparisons played a major role in the genesis of the hypothesis that an excessive intake of salt causes a raised blood pressure (BP) and thence hypertension [5]. The most important evidence on the relationship between sodium intake and BP has come from RCTs. The findings have firmly established that a reduced sodium intake leads to a lowering of BP [6].

There has also been an enormous amount of research into the body mechanisms that regulate BP. However, there is no good reason to believe that this research has usefully contributed to our understanding of how much impact salt intake has on BP.

\section{Example 2: Sugar-sweetened beverages, dietary fat, and body weight}

There has been much research into whether sugar-sweetened beverages (SSBs) and dietary fat induce overconsumption of food energy and thence lead to overweight and obesity. Many cohort studies and RCTs have been conducted and have generated a wealth of valuable information.

Most cohort studies that have investigated SSBs have reported that persons with a relatively high intake of these foods are at increased risk of greater weight gain and hence obesity [7]. RCTs have also indicated this: increased intake of SSBs leads to greater weight gain, while, conversely, replacing SSBs with noncaloric beverages has the opposite effect [7].

Only about one third of cohort studies on adults reported a positive association between dietary fat and subsequent weight while most studies failed to detect an association [8,9]. Many RCTs have also been carried out. These have been of two distinct types. In some, overweight or obese subjects were instructed to follow a calorie-reduced diet in order to help achieve weight loss. Studies with this design have reported that varying the proportion of energy coming from dietary fat has minimal effect on weight loss [10]. Other RCTs have been carried out where weight loss was not a goal. Subjects were instructed to consume either a low-fat or a high-fat diet but were not instructed to reduce energy intake. These RCTs consistently observed that a diet with a reduced fat content leads to spontaneous weight loss (or a slower rate of weight gain) [9]. In summary, the various studies on the relationship between dietary fat and weight gain have reported findings that are much weaker and less consistent than was the case with the findings on SSBs. A reasonable conclusion is that dietary fat has a modest causal relationship with body weight which is of a lower magnitude than that seen with SSBs.

There has also been much research into the pathophysiological mechanisms that lead to obesity, such as the role of leptin and ghrelin. Very little has emerged from this body of mechanistic research that provides useful indications of whether changing the dietary intake of SSBs or fat (or of other aspects of the diet) is likely to affect body weight. Clearly, mechanistic research has generated far less useful information than has come from cohort studies and RCTs.

\section{Example 3: Selenium and cancer}

There has been considerable interest in the possible protective action of selenium against cancer. The key type of research has been epidemiology (cohort studies and population comparisons) [11-13] and RCTs [14]. While the findings are still not sufficient to allow for definitive conclusions, much valuable information has accumulated. Further research along these lines will likely tell us how selenium can be used for the chemoprevention of cancer (dose, types of subjects).

There has also been much research into the mode of action of selenium at the cellular level; some of this is relevant to how the mineral may influence the development of cancer. Rayman [15] carried out a detailed review of the possible role of selenium in the prevention of cancer. She identified nine distinct mechanisms by which this may occur, each of which is a complicated story. Because of this great complexity it is extremely difficult to see how this work contributes information of practical value.

\section{Example 4: Trans fatty acids and coronary heart disease (CHD)}

Several cohort studies have reported that trans fatty acids are associated with an increased risk of CHD [16]. Mozaffarian et al. [16] reviewed studies that give insights into possible mechanisms that might explain these observations. Trans fatty acids have a variety of effects on the body, including harmful changes to both blood lipids and markers of inflammation. It seems very likely that these effects are important in explaining why trans fatty acids increase the risk of CHD. Having at least a partial understanding of how 
these lipids affect the risk of CHD may be intellectually satisfying and (more importantly) may serve to increase confidence that the associations are truly causal. However, the practical value of this mechanistic research is debateable. This is because, first, the mechanisms of atherogenesis are complex, and, second, the role of trans fatty acids is likely to be considerably more complicated than our present knowledge suggests. For these reasons, therefore, another large, well-conducted cohort study is likely to be of considerably more value than further mechanistic research.

\section{Example 5: Fish, fish oil, and CHD}

This example is remarkably similar to the previous one, except that in this case the dietary component is protective against CHD. A great deal of research has been carried out on the possible value of fish and fish oil in the prevention of heart disease. Based on the findings from numerous cohort studies it is now reasonably well established that regular consumption of fish, especially fatty fish, significantly reduces risk of CHD mortality [17]. RCTs (mostly secondary prevention studies) have shown that fish oil modestly prevents risk of heart disease, especially cardiac death; this was of borderline significance [18].

Mozaffarian and $\mathrm{Wu}$ [19] reviewed numerous possible mechanisms that might explain these observations. It is generally accepted that the main component of fish and fish oil responsible for the cardioprotective benefit is n-3 polyunsaturated fatty acid (PUFA). These fats have many effects on the body but it is still far from clear which ones are key with regard to the prevention of CHD. Similar arguments made above with respect to trans fatty acids apply here: the etiology of heart disease is complex, as is the role of n-3 PUFA. Therefore, yet again, the practical value of this mechanistic research is questionable.

\section{Example 6: Meat, total mortality, cardiovascular disease, and cancer}

Several cohort studies have reported that consumption of processed meat is associated with an increased risk of mortality from cardiovascular disease (CVD) and cancer [20-23]. Processed meat is also associated with higher all-cause mortality. A similar association has been reported for red meat [20,21] but there is a lack of consistency in the findings [22,23].

The contrast with the previous example is informative. There is a consensus regarding the component of fish that has the cardioprotective action, yet mechanistic research is still far from being able to identify the key mechanisms(s) of action with any confidence. With meat, by contrast, there is only speculation regarding which components are responsible for increasing the risk of CVD and cancer. Accordingly, we cannot be confident that mechanistic research will be able to explain this, at least not within the next two or three decades. It follows, therefore, that the research strategy that is most likely to generate valuable information is the conduct of more large, well-conducted cohort studies, possibly combined with RCTs where meat eaters switch to a meatless diet.

\section{Example 7: Fruit, vegetables, whole grains, CHD, and cancer}

This example is similar to the previous one except that the foods in question are protective against disease rather than increasing risk. Cohort studies have revealed that a generous intake of fruit and vegetables reduces the risk of cancer, though the magnitude of this is not clear [24-26]. These foods are also protective against CHD [22]. Cohort studies have also demonstrated a clear inverse association between intake of whole grains and risk of both colon cancer [27] and CHD [22].

For the same reason as in the previous example mechanistic research is unlikely to lead to significant contributions in this area. By far the best bet for major progress is the launching of more cohort studies and RCTs.

\section{Looking More Closely at Mechanistic Studies}

It seems almost counter-intuitive to propose that mechanistic studies are of limited value. After all our understanding of disease etiology has undeniably made enormous progress over the past several decades. However, an objective assessment compels the conclusion that real progress in achieving success against disease - meaning understanding how to take measures of practical value that allow disease to be prevented or effectively treated - has been painfully slow. Why is this?

Body processes that lead to disease can best be viewed as a block box. Factors that play a role in either causing or prevent disease (such as genetics and diet) lead into the box on one side. At some later time disease emerges on the other side. With most diseases the black box contains complex mechanisms. In these cases, advances of practical value are most often achieved by focusing on whether the factors leading into the black box affect the outcome, while paying little attention to the actual inner workings of the box.

The degree of complexity of disease processes covers a wide range. At one end of the spectrum simple mechanisms are involved. In those cases studying the mechanisms can be of much value. Several metabolic disorders with a genetic basis illustrate this; for example, PKU, galactosemia, and lactose intolerance. This also applies to many types of vitamin and mineral deficiency. More often, though, the disease process is far more complex, as exemplified by the diseases discussed earlier in this paper. In these cases it is therefore extremely difficult to explain how diet affects disease risk in terms of the effects of food components on disease mechanisms. Carcinogenesis lies at the far end of the spectrum as the disease process is enormously complex. The same applies to genomic medicine. A vast research endeavor is presently underway that is intended to learn everything about human DNA, genomic variations, and their relationship to disease etiology. A goal is to create a personalized genomic medicine that will revolutionize medicine. Based on the above arguments it can be predicted that this work is doomed to achieve little as it massively underestimates the extent of biologic complexity [28,29]. 


\section{Research on Phytochemicals}

Fruit, vegetables, and whole grains contain a great variety of bioactive substances, many of which are likely to play a role in protection against $\mathrm{CHD}$, cancer, and other diseases. One group that is being actively researched is phytochemicals. Based on the arguments made here it follows that the most appropriate research strategy is one centered on cohort studies and RCTs [30]. By contrast, a research strategy that attempts to, first, develop an understanding of the role played by individual phytochemicals in metabolism and cellular function, and then to translate this information into practical nutritional advice on preventing or treating disease is unlikely to produce useful information. Despite the serious limitations of that research strategy a great deal of mechanistic research is presently being carried out on phytochemicals. In a recent review Cherniak [31] discussed several dozen mechanistic studies that investigated the effects of phytochemicals or phytochemical-rich plant extracts on risk of CVD or the metabolic syndrome. Adding to the questionable value of this research, much of it was carried out on experimental animals.

In 1927 Werner Heisenberg published what is known as Heisenberg's uncertainty principle [32]. Put simply this states that the more precisely the position of an electron or any other particle is measured, the less precisely can its momentum be measured. The arguments made above suggest that a similar rule holds true for phytochemicals: the more precisely one analyzes their biochemical actions, the less accurately can one understand their effects on health.

\section{Conclusion}

The examples examined here reveal that most of our information of practical value in the area of nutrition in relation to health and disease has come from cohort studies and RCTs. By contrast, relatively little of it has come from mechanistic research. These lessons should be applied to the planning of future research studies. Accordingly, research resources should be focused on carrying out more cohort studies and RCTs.

\section{References}

1. Roberts S, Temple NJ (2002) Medical research: a bettor's guide. Am J Prev Med 23: 231-2.

2. Temple NJ (1994) Medical research: a complex problem. In: Temple NJ, Burkitt DB, eds. Western diseases: their dietary prevention and reversibility. Humana Press, New Jersey 419-36.

3. Temple NJ (2002) Nutrition and disease: challenges of research design. Nutrition 18: 343-7.

4. Jacobs DR, Temple NJ (2012) Methods in nutrition research. In: Temple NJ, Wilson T, Jacobs DR, editors. Nutritional health: strategies for disease prevention, 3rd ed. Humana Press, New York 1-27.

5. Elliott P, Stamler J, Nichols R, Dyer AR, Stamler R, et al. (1996) Intersalt revisited: further analyses of 24 hour sodium excretion and blood pressure within and across populations. BMJ 312: 1249-53.

6. He FJ, Li J, Macgregor GA (2013) Effect of longer term modest salt reduction on blood pressure: Cochrane systematic review and meta-analysis of randomized trials. BMJ 346: f1325

7. Malik VS, Pan A, Willett WC, Hu FB (2013) Sugar-sweetened beverages and weight gain in children and adults: a systematic review and meta-analysis. Am J Clin Nutr 98: 1084-102.

8. Forouhi NG, Sharp SJ, Du H, Van der A DL, Halkjaer J, et al. (2009) Dietary fat intake and subsequent weight change in adults: results from the European Prospective Investigation into Cancer and Nutrition cohorts. Am J Clin Nutr 90: 1632-41.

9. Hooper L, Abdelhamid A, Moore HJ, Douthwaite W, Skeaff CM, et al. (2012) Effect of reducing total fat intake on body weight: systematic review and metaanalysis of randomised controlled trials and cohort studies. BMJ 345: e7666.

10. Sacks FM, Bray GA, Carey VJ, Smith SR, Ryan DH, et al. (2009) Comparison of weight-loss diets with different compositions of fat, protein, and carbohydrates. N Engl J Med 360: 859-73.

11. Etminan M, FitzGerald JM, Gleave M, Chambers K (2005) Intake of selenium in the prevention of prostate cancer: a systematic review and meta-analysis. Cancer Causes Control 16: 1125-31.

12. Zhuo H, Smith AH, Steinmaus C (2004) Selenium and lung cancer: a quantitative analysis of heterogeneity in the current epidemiological literature. Cancer Epidemiol Biomarkers Prev 13: 771-8.

13. Schrauzer GN, White DA, Schneider CJ (1977) Cancer mortality correlation studies--III: statistical associations with dietary selenium intakes. Bioinorg Chem 7:23-31.

14. Lee EH, Myung SK, Jeon YJ, Kim Y, Chang YJ, et al. (2011) Effects of selenium supplements on cancer prevention: meta-analysis of randomized controlled trials. Nutr Cancer 63: 1185-95.

15. Rayman MP (2005) Selenium in cancer prevention: a review of the evidence and mechanism of action. Proc Nutr Soc 64: 527-42.

16. Mozaffarian D, Katan MB, Ascherio A, Stampfer MJ, Willett WC (2006) Trans fatty acids and cardiovascular disease. N Engl J Med 354: 1601-13.

17. Zheng J, Huang T, Yu Y, Hu X, Yang B, et al. (2012) Fish consumption and CHD mortality: an updated meta-analysis of seventeen cohort studies. Public Health Nutr 15: 725-37.

18. Rizos EC, Ntzani EE, Bika E, Kostapanos MS, Elisaf MS (2012) Association between omega-3 fatty acid supplementation and risk of major cardiovascular disease events: a systematic review and meta-analysis. JAMA 308: 1024-33.

19. Mozaffarian D, Wu JH (2011) Omega-3 fatty acids and cardiovascular disease: effects on risk factors, molecular pathways, and clinical events. J Am Coll Cardiol 58: 2047-67.

20. Sinha R, Cross AJ, Graubard BI, Leitzmann MF, Schatzkin A (2009) Meat intake and mortality: a prospective study of over half a million people. Arch Intern Med 169: 562-71.

21. Pan A, Sun Q, Bernstein AM, Schulze MB, Manson JE, et al. (2012) Red meat consumption and mortality: results from 2 prospective cohort studies. Arch Intern Med 172: 555-63.

22. Mozaffarian D, Appel LJ, Van Horn L (2011) Components of a cardio protective diet: new insights. Circulation 123: $2870-91$.

23. Rohrmann S, Overvad K, Bueno-de-Mesquita HB, Jakobsen MU, Egeberg R, et al. (2013) Meat consumption and mortality--results from the European Prospective Investigation into Cancer and Nutrition. BMC Med 11: 63. 
24. Riboli E, Norat T (2003) Epidemiologic evidence of the protective effect of fruit and vegetables on cancer risk. Am J Clin Nutr 78: 559S-69S.

25. Boffetta P, Couto E, Wichmann J, Ferrari P, Trichopoulos D, et al. (2010) Fruit and vegetable intake and overall cancer risk in the European Prospective Investigation into Cancer and Nutrition (EPIC). J Natl Cancer Inst 102: 529-37.

26. Hung HC, Joshipura KJ, Jiang R, Hu FB, Hunter D, et al. (2004) Fruit and vegetable intake and risk of major chronic disease. J Natl Cancer Inst 96: 1577-84. 27. Aune D, Chan DS, Lau R, Vieira R, Greenwood DC, et al. (2011) Dietary fibre, whole grains, and risk of colorectal cancer: systematic review and dose-response meta-analysis of prospective studies. BMJ 343: d6617.

28. Baird PA, Temple NJ (2007) Genetics, genomic medicine, and achieving better population health: a flawed strategy. In: Temple NJ, Thompson A, eds. Excessive medical spending: facing the challenge. Radcliffe Publishing, Oxford 110-11.

29. McGuire AL, Cho MK, McGuire SE, Caulfield T (2007) The future of personal genomics. Science 317: 1687.

30. Temple NJ (2012) What is the optimum research strategy to study the effects of phytochemicals on disease prevention? Nutrition $28: 611-2$.

31. Cherniack EP (2011) Polyphenols: planting the seeds of treatment for the metabolic syndrome. Nutrition 27: 617-23.

32. Wikipedia. 2014. Uncertainty principle. 